\title{
Transarterial oily chemoembolization with lidamycin shows potent therapeutic efficacy in VX2 rabbit liver tumor
}

\author{
Genshen Zhongl,* \\ Jinsong $\mathrm{Qi}^{2}$,* \\ Shuhua Huo' \\ Huichao Xue ${ }^{3}$ \\ Zhishan $\mathrm{Xu}^{\mathrm{I}}$ \\ Jinsong $\mathrm{Li}^{4}$ \\ Yanjun Zhou ${ }^{5}$ \\ Minna Wu' \\ Liang $\mathrm{Li}^{6}$
}

'Laboratory of Cancer Biotherapy, Institute of Neurology, The First Affiliated Hospital of Xinxiang Medical University, Weihui, Henan Province, People's Republic of China; ${ }^{2}$ Department of Intervention, ${ }^{3}$ Department of General Surgery,

${ }^{4}$ Department of Pathology,

${ }^{5}$ Department of Clinical Laboratory, The First Affiliated Hospital of Xinxiang Medical University, Weihui, Henan Province, People's Republic of China; ${ }^{6}$ nstitute of Medicinal Biotechnology, Chinese Academy of Medical Sciences and Peking Union Medical College, Beijing, People's Republic of China

*These authors contributed equally to this work
This article was published in the following Dove Press journal:

OncoTargets and Therapy

22 October 2015

Number of times this article has been viewed
Abstract: Transarterial oily chemoembolization (TOCE) is one of the most effective approaches for the treatment of patients with hepatocellular carcinoma (HCC), who are not suitable for surgical therapy. Lidamycin (LDM), a potent antitumor antibiotic, demonstrates good antitumor efficacy in various tumor types, both in vitro and in vivo. In this study, the antitumor efficacy of LDM combined with TOCE against the rabbit VX2 tumor was assessed. A toxicity assay with 3-(4,5-dimethylthiazol-2-yl)-2,5-diphenyltetrazolium bromide (MTT) demonstrated that a combination of LDM with lipiodol did not impair the cytotoxicity of LDM against HepG2 cells in vitro. Using TOCE in rabbit VX2 tumor models, LDM showed a more powerful inhibitory effect against the tumor and lowered the expression levels of proliferating cell nuclear antigen (PCNA), cluster of differentiation 31 (CD31), and vascular endothelial growth factor (VEGF) compared to Adriamycin (ADM); moreover, this improvement was not accompanied by an increase of hepatotoxicity as shown by alanine aminotransferase (ALT) and aspartate aminotransferase (AST) levels. These results suggested that LDM combined with TOCE may be a feasible strategy in HCC therapy in the future.

Keywords: lidamycin, TOCE, hepatocellular carcinoma, VX2, Adriamycin

\section{Introduction}

Hepatocellular carcinoma $(\mathrm{HCC})$ is one of the most common malignancies worldwide. ${ }^{1}$ At the present time, the conventional treatment method for HCC is surgical excision, chemotherapy, and radiotherapy. ${ }^{2,3}$ An operation can reduce the tumor load and has resulted in clear benefits; however, only $10 \%-30 \%$ of patients with HCC qualify for surgical treatment because of additional complications, such as liver dysfunction, cirrhosis, and multiple lesions of the tumor foci. ${ }^{4}$ Moreover, many patients with HCC are diagnosed in the advanced stage, when surgical treatment is no longer an option. ${ }^{5,6}$ The therapeutic efficacy of chemotherapy via intravenous injection is often not considered due to the low intratumoral drug concentration resulting from dilution in the blood, inevitably accompanied by severe systemic chemotherapeutic side effects, such as hair loss, nausea, vomiting, and bone marrow suppression. ${ }^{7}$ Local radiotherapy as a treatment option for $\mathrm{HCC}$ is subject to limitations due to the invisible foci or tumors located near large blood vessels in the liver. ${ }^{8}$ In addition, the effective dosage of radiation must be larger than $60 \mathrm{~Gy}$; however, the tolerated radiotherapy dosage of normal liver tissue is less than $40 \mathrm{~Gy} .{ }^{9}$ Therefore, radiotherapy for $\mathrm{HCC}$ is not preferred in the clinical setting. Currently, the reported 5-year survival rate for patients with HCC is far from satisfactory and the question of how to improve the clinical efficacy of HCC treatments needs further exploration.
Correspondence: Genshen Zhong Laboratory of Cancer Biotherapy, Institute of Neurology, The First Affiliated Hospital of Xinxiang Medical University, Jiankang Road 88, Weihui 453100, Henan Province, People's Republic of China Tel +86373440 4277

Email zhonggs@xxmu.edu.cn 
Treatment of HCC using transarterial oily chemoembolization (TOCE) has gained some acceptance due to its advantages of being minimally invasive, yielding a high local drug concentration, blocking the blood supply to the tumor, the low systemic side effects etc. ${ }^{10,11}$ Drugs often used in TOCE include Adriamycin (ADM), mitomycin, cisplatin, and 5 -fluorouracil, ${ }^{12}$ which are given in a mixed formation with lipiodol to treat progressive HCC. Compared with systemic chemotherapy via intravenous injection, TOCE may increase the intratumoral drug concentration and reduce systemic adverse reactions. Lipiodol acts as a carrier and embolic agent, and can embolize small arteries and block peripheral vascular blood flow, thus generating a microenvironment of tumor hypoxia and ischemia. However, studies have found that the cytotoxicities of many antitumor drugs may be reduced under hypoxic conditions. ${ }^{13}$ At the same time, drug resistance may be induced after the repeat usage of chemotherapy. ${ }^{14}$ All this could lead to a decrease of the antitumor activity and a lower clinical efficacy. In order to improve the clinical efficacy of $\mathrm{HCC}$, it is necessary to find new, alternative agents.

Lidamycin (LDM; also known as C-1027) is a potent antitumor antibiotic. Its in vitro activity is approximately 1,000 times higher than that of ADM, which is commonly used in the clinic. ${ }^{15}$ A study has shown that the expression of a multidrug resistance gene in cancer cells had little effect on the activity of LDM. ${ }^{16}$ Furthermore, the antitumor activity of LDM could be increased approximately threefold under hypoxic conditions. ${ }^{17} \mathrm{LDM}$ is currently in Phase II clinical trials (registration number: CN2006L03713) and has good application prospects. In this study, the antitumor efficacy of LDM on a VX2 rabbit tumor using the TOCE method was investigated so as to provide pharmacological evidence for clinical HCC therapy with LDM via an interventional approach.

\section{Materials and methods Ethics statement}

The animal experiments were approved by the Ethics Committee of The Affiliated Hospital of Xinxiang Medical University, and carried out in accordance with a protocol approved by our hospital animal care and use committee and in compliance with institutional guidelines.

\section{Cell culture and toxicity assays}

Human hepatoma HepG2 cells were grown in Roswell Park Memorial Institute (RPMI-1640; Gibco, Gaithersburg, MD, USA) media supplemented with $10 \%$ fetal bovine serum (Gibco), penicillin-streptomycin (1\%), and $2 \mathrm{mML}$-glutamine. For the toxicity assays with 3-(4,5-dimethylthiazol-2-yl)-2,5- diphenyltetrazolium bromide (MTT), HepG2 cells were detached by trypsinization and seeded at 5,000 cells/well in a 96-well plate (Costar, Washington, DC, USA) overnight. Then different concentrations of LDM (1 nM), lipiodol $(0.1 \% \mathrm{v} / \mathrm{v})$, and a mixture of LDM with lipiodol were added to separate wells and the cells were incubated for an additional 24 hours. The effects on cell growth were examined as described previously. ${ }^{18}$

\section{Animal models}

Twenty-two New Zealand white rabbits (male, weight 3.0-3.5 $\mathrm{kg}$ ) were purchased from the experimental animal center of Xinxiang Medical University. VX2 carcinoma cells were purchased from the animal center of Sun Yat-sen University and then maintained as a tumor line in our laboratory. The rabbits (20 rabbits were used) were anesthetized via a marginal ear vein injection with sodium pentobarbital $(25 \mathrm{mg} / \mathrm{kg})$. Then, the hair over the abdominal region was removed with $8 \%$ sodium sulfide and the region was further cleaned with iodophor. To obtain a suspension of VX2 tumor cells, a cryopreserved VX2 tumor was first inoculated into the right hind leg muscle of a carrier rabbit (two carrier rabbits were used). The tumor was allowed to grow for approximately 2 weeks and then dissected and cut into small pieces $(2 \mathrm{~mm} \times 2 \mathrm{~mm} \times 2 \mathrm{~mm})$. Then, the VX2 tumor tissue $\left(\sim 2-2.5 \times 10^{6}\right.$ cells $)$ was injected via an 18 -gauge needle into the left lobe of the liver of a study rabbit percutaneously under ultrasound guidance. At the same time, penicillin $(100,000 \mathrm{IU} / \mathrm{kg})$ was injected intramuscularly after the implantation, and this injection was repeated for 3 additional days. The transplanted VX2 tumors were observed by sonography until the tumors reached $1 \mathrm{~cm}$ in diameter, and the ultrasonography was performed by the same operator each time. Rabbits were then randomly assigned to one of two groups ( $\mathrm{n}=10$ per group). One group received $1 \mathrm{~mL}$ of a mixed preparation of lipiodol with $\operatorname{ADM}(2 \mathrm{mg} / \mathrm{kg})$, while the other group received $1 \mathrm{~mL}$ of a mixed preparation of lipiodol with LDM $(100 \mu \mathrm{g} / \mathrm{kg})$.

\section{Arterial catheterization}

Rabbits were anesthetized via a marginal ear vein injection with sodium pentobarbital $(25 \mathrm{mg} / \mathrm{kg})$ and laid down on the table of a digital subtraction angiography (DSA) machine (Toshiba INFX-8000C, Toshiba, Tokyo, Japan). An incision was made to expose the right femoral artery. Then a microcatheter (EchelonTM-14 micro-catheter, Micro Therapeutics Inc. dba ev3 Neurovascular Terumo, Irvine, CA, USA) was inserted into the femoral artery and positioned into the hepatic artery under the guidance of fluoroscopy. 
Next, the perfusion preparation (a mixture of LDM [100 $\mu \mathrm{g} / \mathrm{kg}$ ] or ADM [2 mg/kg] with lipiodol) at a total volume of $1 \mathrm{~mL}$ for each rabbit was gradually hand-injected into the hepatic artery under fluoroscopy guidance, with care taken regarding the guidewire to keep the catheter tip in the hepatic artery to avoid efflux of the preparation. After intraarterial injection, the catheter was removed and the femoral artery was sutured; as a tiny incision was made in the femoral artery during TOCE and then sutured after surgery, and we did not experience any specific problems. DSA images of VX2 liver tumor staining were acquired before and after the infusion.

\section{Animal sacrifice}

After infusion of the preparation for 3 days, three rabbits from each group were sacrificed via an overdose of sodium pentobarbital $(25 \mathrm{mg} / \mathrm{kg})$ and the tumor tissues were collected for Western blot and immunohistochemistry (IHC) analysis. At day 10 after the infusion, the remaining seven rabbits in each group were sacrificed after ultrasonography monitoring of the tumor size. The livers were excised and the tumors were measured to give the lengths $(a)$ and the widths $(b)$ via incision of the liver, and the tumor volumes were calculated using the formula: $0.5 \times a b^{2}$.

\section{$\mathrm{IHC}$}

Parts of the VX2 tumor samples collected from the rabbits were fixed in $10 \%$ formalin and embedded in paraffin. Slices (thickness $5 \mu \mathrm{m}$ ) were cut and processed for IHC staining. The other parts of the VX2 tumor samples were used for protein extraction and Western blot analyses.

To detect the expression and location of proliferating cell nuclear antigen (PCNA), vascular endothelial growth factor (VEGF), cluster of differentiation 31 (CD31), matrix metalloproteinase 2 (MMP2), and MMP9 in tumor tissues, the sample sections were incubated with mouse anti-PCNA, anti-VEGF, anti-MMP-2, anti-MMP-9, and anti-CD31 (Abcam, Cambridge, UK) monoclonal antibodies overnight and then with anti-mouse secondary antibodies (Beyotime, Shanghai, People's Republic of China) for 45 minutes at room temperature, after which the slides were visualized with 3-3'-diaminobenzidine tetrahydrochloride (DAB) substrate. Hematoxylin was used to counterstain the nuclei of the tumor cells. The results of the IHC were evaluated by a pathologist in The First Affiliated Hospital of Xinxiang Medical University in a blinded manner via light microscopy. The protein expression levels were analyzed by Image-Pro Plus software (Media Cybernetics, Bethesda, MD, USA), and the results are represented as the mean \pm standard deviation (SD) of five high performance fields.

\section{Western blotting}

The samples were lysed in ice-cold lysis buffer containing $2 \mathrm{mM}$ ethylenediaminetetraacetic acid (EDTA), $20 \mathrm{mM}$ Tris- $\mathrm{HCl}, 1 \%$ Triton $\mathrm{X}-100$, and a protease inhibitor mix $(\mathrm{pH}$ 7.5) using a glass homogenizer. The protein concentrations were determined using a BCA protein assay (Pierce, Rockford, IL, USA). The proteins were separated by sodium dodecyl sulfate-polyacrylamide gel electrophoresis (SDS-PAGE) and transferred to polyvinylidene fluoride (PVDF) membranes (Millipore, Bedford, MA, USA). An anti-hypoxia-inducible factor- $1 \alpha$ (anti-HIF-1 $\alpha$ ) antibody (Abcam) and an anti- $\beta$-actin antibody (Santa Cruz, CA, USA) were used as internal controls. Horseradish peroxidase- (HPR-) conjugated secondary antibody was then incubated with the membrane and the blots were developed with a chemiluminescence substrate solution (Millipore, Bedford, MA, USA) and exposed to an X-ray film (Kodak, NY, USA). Image J software was used to calculate the expression levels of protein using $\beta$-actin as a control.

\section{Aspartate aminotransferase and alanine aminotransferase levels}

To evaluate the hepatotoxicity caused by LDM, peripheral blood was sampled from the auricular veins of all animals before the experiment and at days 3 and 10 after intra-arterial administration. The aspartate aminotransferase (AST) and alanine aminotransferase (ALT) levels were determined with a biochemical autoanalyzer (Olympus AU 2700, Olympus, Tokyo, Japan).

\section{Statistical analyses}

All results are shown as the mean \pm SD for each group. Student's $t$-test was performed to compare the two groups, and $P<0.05$ was considered statistically significant. Statistical analyses were performed using SPSS 16.0 for Windows (SPSS Inc., Chicago, IL, USA).

\section{Results}

\section{The influence of iodized oil on the cytotoxicity of LDM in vitro}

In order to exclude the possibility that iodized oil (IDO) influenced the cytotoxicity of LDM in cancer cells, an MTT assay was performed. As shown in Figure 1, IDO with LDM for 24 hours at $4^{\circ} \mathrm{C}$ did not reduce the cytotoxicity of LDM against hepatoma HepG2 cells. The percentage of viable cells 


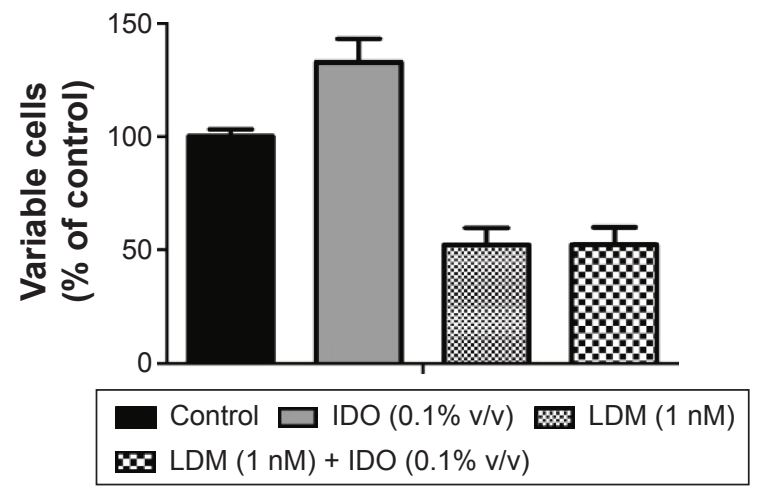

Figure I Cytotoxicity of LDM or LDM combined with IDO on HepG2 cells determined via an MTT assay.

Note: Data represented triple results, mean $\pm S D$.

Abbreviations: LDM, lidamycin; IDO, iodized oil; SD, standard deviation.

following treatment with LDM (1 nM) or LDM (1 nM) mixed with IDO $(0.1 \% \mathrm{v} / \mathrm{v})$ were $52.21 \%$ and $52.26 \%$, respectively, compared with control cells. Thus, mixing LDM with IDO did not reduce the antitumor effect of LDM in vitro. Interestingly, as shown in Figure 1, the number of viable cells in the IDO group was higher than the control, which may suggest that IDO could influence the absorbance of the MTT assay, making the detected value higher than the real value. However, the detected values for the LDM group and the cells treated with LDM combined with IDO had no apparent difference, meaning that LDM combined with IDO was cytotoxic to HepG2 cells in vitro regardless of the influence of IDO on the MTT assay results.

\section{VX2 tumor and transcatheter procedure}

The VX2 tumor was transplanted into the left lobe of the liver of rabbits and detected by ultrasound after 2 weeks. After this time, VX2 tumors were found to be successfully grafted in 20 rabbits (Figure 2). Twenty study rabbits were then randomly divided into two groups: an ADM + IDO group and an LDM + IDO group, whereby the size of the VX2 tumors in the ADM + IDO group and the LDM + IDO group were $344.0 \pm 27.3 \mathrm{~mm}^{3}$ and $357.2 \pm 33.0 \mathrm{~mm}^{3}$ (Table 1), respectively. The transcatheter procedure described in the "Materials and methods" section was carried out successfully in all of the rabbits (Figure 2).
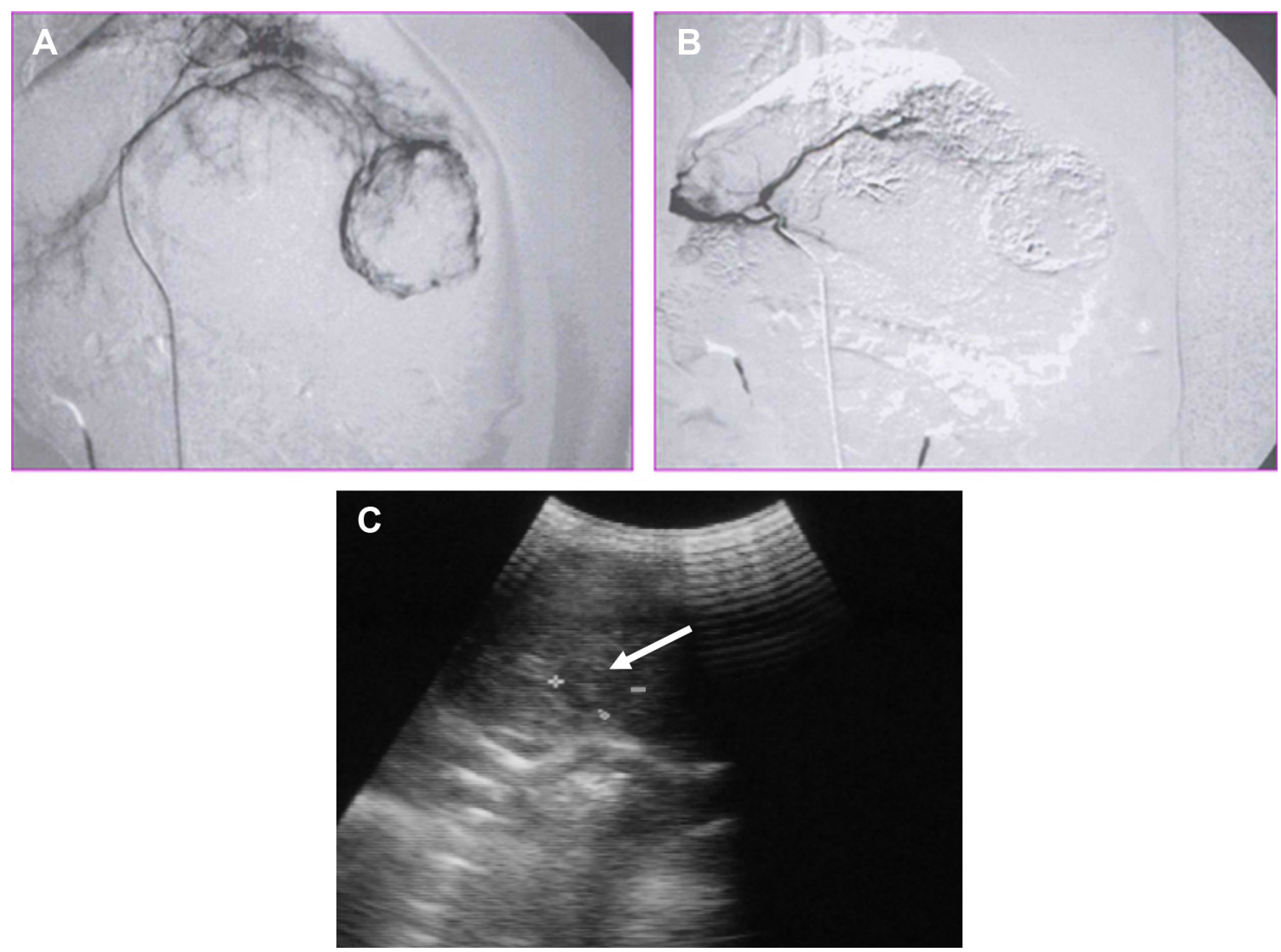

Figure 2 DSA and ultrasound images of a VX2 tumor transplanted in a rabbit liver.

Notes: (A) Angiography of the hepatic artery showing a rich blood supply to the tumor, especially the rim of the tumor. (B) Embolization with IDO, which was deposited well in the lesion. (C) Ultrasound image showing a relative hyperintensity VX2 tumor in the liver lobe (white arrow). The (+) and $(-)$ labels were used to measure the size of the tumor, with the distance between the $(+)$ and $(-)$ labels giving the diameter.

Abbreviations: DSA, digital subtraction angiography; IDO, iodized oil. 
Table I Inhibitory efficacy of ADM + IDO and LDM + IDO on rabbit VX2 tumors

\begin{tabular}{llll}
\hline Group & $\begin{array}{l}\text { Tumor } \\
\text { volume before } \\
\text { infusion }\left(\mathbf{m m}^{3}\right)\end{array}$ & $\begin{array}{l}\text { Tumor volume } \\
\text { at I0 days after } \\
\text { infusion }\left(\mathbf{m m}^{3}\right)\end{array}$ & TGR \\
\hline ADM + IDO & $344.0 \pm 27.3$ & $\mathrm{I}, 774.1 \pm 444.7$ & $4.72 \pm 0.62$ \\
LDM + IDO & $357.2 \pm 33.0$ & $\mathrm{I}, 145.9 \pm 295.2$ & $3.39 \pm 1.13^{*}$ \\
\hline
\end{tabular}

Note: $* P<0.05$, compared to the ADM + IDO group.

Abbreviations: TGR, tumor volume at 10 days after injection/tumor volume before injection; ADM, Adriamycin; IDO, iodized oil; LDM, lidamycin.

\section{Determination of ALT and AST levels}

The ALT and AST levels were determined to evaluate the hepatotoxicity of ADM + IDO and LDM + IDO treatment. As shown in Figure 3, compared with the levels before TOCE surgery,
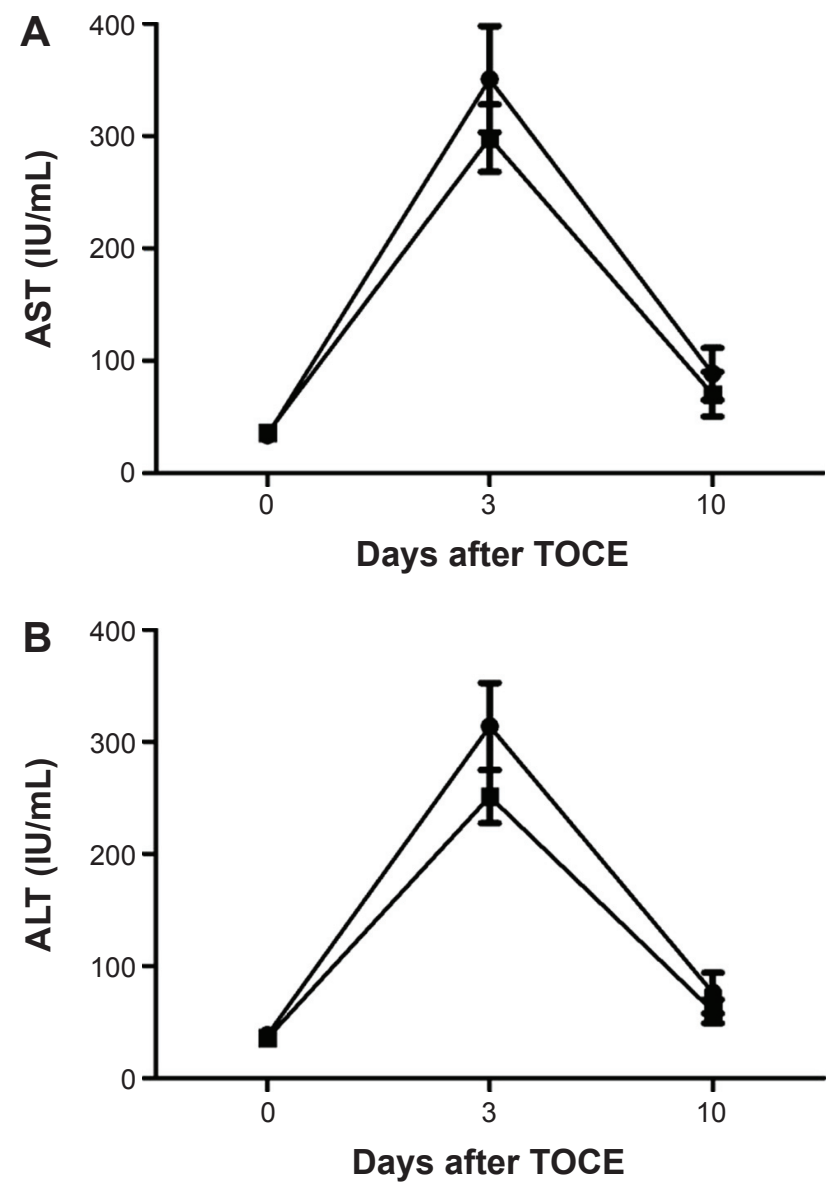

$$
\rightarrow \text { ADM + IDO } \rightarrow \text { LDM + IDO }
$$

Figure 3 Changes in the rabbit serum ALT and AST levels after TOCE.

Notes: Blood chemical analysis suggested that there was an increase in the serum AST (A) and ALT (B) levels in the ADM + IDO and LDM + IDO groups at 3 days after perfusion as compared to those at 0 and 10 days, but there was no significant difference between the ADM + IDO and LDM + IDO groups. The results indicated that the influence of ADM + IDO or LDM + IDO perfusion on rabbit serum ALT and AST levels was transient and recoverable.

Abbreviations: ALT, alanine aminotransferase; AST, aspartate aminotransferase; TOCE, transarterial oily chemoembolization; ADM, Adriamycin; IDO, iodized oil; LDM, lidamycin. the ALT and AST levels in all rabbits transiently increased on the 3rd day after intra-arterial administration. However, the levels were restored to normal on the 10th day after treatment. Differences in the ALT and AST levels after treatment with $\mathrm{ADM}+\mathrm{IDO}$ or LDM + IDO at 0 and 3 days were significant $(P<0.05)$. However, there was no significant difference in the ALT and AST levels between the ADM + IDO and LDM + IDO groups $(P>0.05)$ after TOCE surgery.

\section{Determination of HIF-I $\alpha$}

Western blotting was used to determine the expression levels of HIF- $1 \alpha$ at 3 days after intra-arterial infusion, and normal liver tissue (collected from nontumor liver tissue in TOCE-treated rabbits) was used as a control. As shown in Figure 4, the results demonstrated that TOCE treatment elevated the expression of HIF-1 $\alpha$, which was upregulated $1.65 \pm 0.07$-fold in the ADM + IDO group and $1.53 \pm 0.13$ fold in the LDM + IDO group. There was no significant difference between the ADM + IDO and LDM + IDO groups with regards to HIF-1 $\alpha$ expression.
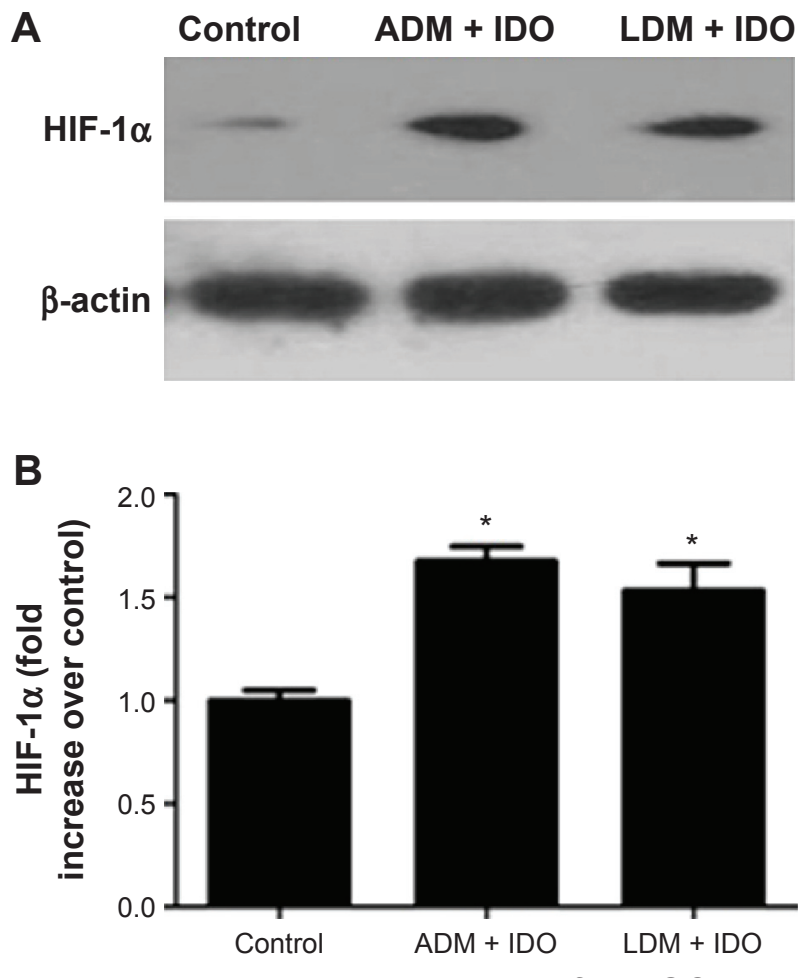

Figure 4 Western blot analysis of HIF- I $\alpha$ expression in rabbit liver tissue. Notes: $(\mathbf{A})$ is the western blot of HIF-I $\alpha$ and (B) was the quantitative analysis of $\mathrm{A}$ using the Image $\mathrm{J}$ software (developed by the National Institutes of Health). Control represents the HIF-I $\alpha$ expression in normal rabbit liver tissue, while ADM + IDO and LDM + IDO represent the HIF-I $\alpha$ expression in VX2 tumor tissues that were collected at day 3 after TOCE with ADM + IDO or LDM + IDO, respectively. There was a significant difference between the ADM + IDO and LDM + IDO groups compared with the control group. The data indicated that HIF-I $\alpha$ was upregulated in rabbit VX2 tumor tissue after TOCE. $* P<0.05$, compared to the control.

Abbreviations: HIF-I $\alpha$, hypoxia-inducible factor-I $\alpha$; ADM, Adriamycin; IDO, iodized oil; LDM, lidamycin; TOCE, transarterial oily chemoembolization. 

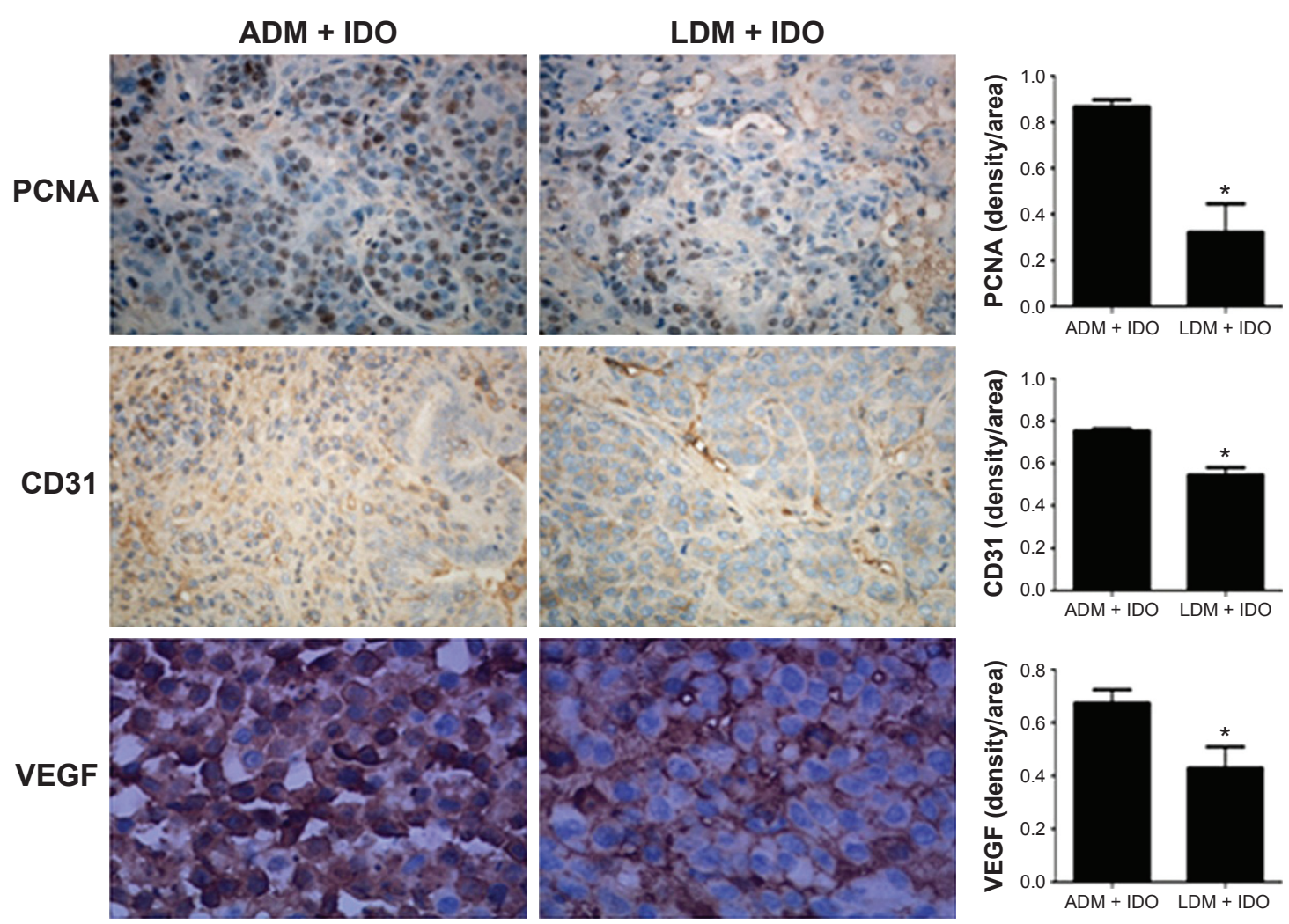

Figure 5 Immunohistochemistry analysis of VX2 tumor sections.

Notes: Effects of ADM + IDO and LDM + IDO on tumor proliferation assessed via PCNA levels, tumor microvessel density assessed via CD3I levels, and tumor angiogenesis assessed via VEGF levels in paraffin-embedded VX2 tumor sections. The IHC analysis of PCNA, CD3I, and VEGF expression indicated the enhanced inhibition of cell proliferation and angiogenesis in the LDM + IDO group compared to the ADM + IDO group. ${ }^{*}<<0.05$.

Abbreviations: ADM, Adriamycin; IDO, iodized oil; LDM, lidamycin; PCNA, proliferating cell nuclear antigen; CD3I, cluster of differentiation 3 I; VEGF, vascular endothelial growth factor; IHC, immunohistochemistry.

\section{IHC analysis of PCNA,VEGF, CD3I, MMP-2, and MMP-9 expression}

IHC was used to detect the expressions of PCNA, VEGF, CD31, MMP-2, and MMP-9, as shown in Figure 5. Compared with the ADM + IDO group, there were significant differences among the expression levels of PCNA, VEGF, and CD31 when cells were treated with LDM + IDO; however, there were no significant differences in the levels of MMP-2 and MMP-9 between the groups (data not shown).

\section{Antitumor efficacy}

To detect the antitumor efficacy of ADM and LDM via TOCE in VX2 rabbit liver tumors, the length and width of the VX2 tumors were measured and used to calculate the volumes of the tumors. As shown in Figure 6, the median tumor volumes of the ADM + IDO and LDM + IDO groups were 1,774 \pm 444 and $1,145 \pm 295 \mathrm{~mm}^{3}$, respectively, showing that the antitumor efficacies of ADM + IDO and LDM + IDO were significantly different $(P<0.05)$, with LDM + IDO resulting in decreased growth of the tumor.

\section{Discussion}

In this study, the antitumor efficacy of LDM on VX2 rabbit liver cancer was evaluated using the TOCE approach. This is the first time that the antitumor efficacy of LDM has been investigated via intra-arterial treatment, as previous

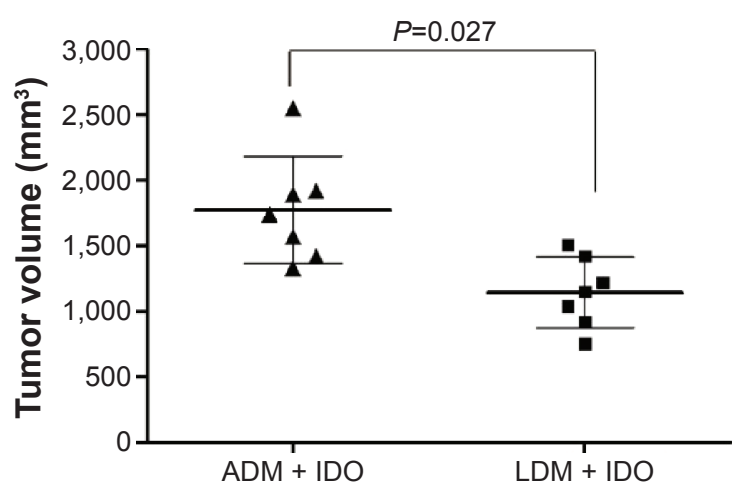

Figure 6 Volume of tumor following treatment with ADM + IDO or LDM + IDO at day 10 after TOCE.

Note: $P=0.027$ for the LDM + IDO group compared with the ADM + IDO group. Abbreviations: ADM, Adriamycin; IDO, iodized oil; LDM, lidamycin; TOCE, transarterial oily chemoembolization. 
studies have used intravenous injection. First, the influence of lipiodol on LDM was investigated using an MTT assay. As shown in Figure 1, the results showed that the mix of LDM and lipiodol did not result in a decrease of cytotoxicity toward tumor cells.

TOCE in rabbits transplanted with VX2 tumors was carried out with a catheter wire as a neural intervention to guarantee the success rate of the procedure. As shown in Figure 2, the DSA image showed that the VX2 tumor was successfully transplanted in the rabbit liver and was then embolized by lipiodol. Lipiodol is an IDO that is commonly used as a vehicle due to its characteristics of selective accumulation at the site of hypervascular hepatic tumors and its prolonged retention after intra-arterial infusion. Thus, lipiodol may act as an agent for the sustained release of drugs, while creating hypoxic conditions at the local site of embolization, which may accelerate the death or apoptosis of tumor cells. However, it should be noted that hypoxic conditions generated by lipiodol infusion could induce the upregulation of HIF- $1 \alpha \cdot .^{19,20}$ It has been documented that the expression of HIF-1 $\alpha$ may induce angiogenesis, ${ }^{21,22}$ drug resistance, ${ }^{23}$ and cancer metastasis. ${ }^{24,25}$ As shown in Figure 4, HIF-1 $\alpha$ expression was upregulated by approximately 1.6-fold after lipiodol infusion.

ADM is an anticancer agent commonly used in the clinic, and its combination with lipiodol may improve the survival rate of HCC patients. In this study, ADM was used as a control drug and the potential of LDM in HCC therapy using the TOCE method was assessed in comparison to ADM. As shown in Figure 5, the expression levels of PCNA, CD31, and VEGF following treatment with LDM + IDO were lower than after ADM + IDO. It is known that PCNA is related to the capability of cell proliferation, while CD31 is correlated with microvessel density and VEGF is associated with vascular angiogenesis. Therefore, their lower expressions suggest a better therapeutic outcome. The VX2 tumor treated with LDM showed lower expressions of PCNA, CD31, and VEGF than the ADM group, indicating that the anticancer efficacy of LDM was better than that of ADM in this cancer type. This was confirmed by the tumor volume, which was measured 10 days after TOCE. The tumor volume following LDM + IDO was $1,145 \pm 295 \mathrm{~mm}^{3}$, while it was $1,774 \pm 444 \mathrm{~mm}^{3}$ for the ADM + IDO group $(P=0.027)$, indicating that LDM + IDO reduced tumor growth.

As shown in Figure 3, the improved antitumor efficacy was not accompanied by an increase of hepatotoxicity, as reflected by the AST and ALT levels, which were similar between the treatment groups. In this study, we also found that there was no significant difference in the expression levels of MMP-2 and MMP-9 between the ADM + IDO and LDM + IDO groups, an explanation for which may be their relatively stable expression, meaning they were not easily shifted by drug treatment.

In conclusion, this study investigated the antitumor efficacy of LDM via hepatic intra-arterial infusion combined with embolization with lipiodol. The results showed that LDM demonstrated better tumor inhibition efficacy than ADM without an increase of hepatotoxicity, as reflected by serum AST and ALT levels. Thus, LDM may be a promising agent for use in unresectable HCC therapy. Further studies on the mechanism, biodistribution, and pharmacokinetics of LDM combined with lipiodol administered via intra-arterial infusion are required to guarantee its clinical application in HCC TOCE therapy.

\section{Acknowledgments}

This study was supported by grants from the National Natural Science Foundation of China (Number 81201765), the Major Science and Technology Projects of China (Number 2013ZX09102064), and the Key Scientific Research Projects in Henan Higher Education College (15A320063).

\section{Disclosure}

The authors report no conflicts of interest in this work.

\section{References}

1. Bosetti C, Turati F, La Vecchia C. Hepatocellular carcinoma epidemiology. Best Pract Res Clin Gastroenterol. 2014;28(5):753-770.

2. Wang Z, Zhang G, Wu J, Jia M. Adjuvant therapy for hepatocellular carcinoma: current situation and prospect. Drug Discov Ther. 2013;7(4): 137-143.

3. Cormier JN, Thomas KT, Chari RS, Pinson CW. Management of hepatocellular carcinoma. J Gastrointest Surg. 2006;10(5):761-780.

4. Osaki Y, Nishikawa H. Treatment for hepatocellular carcinoma in Japan over the last three decades: our experience and published work review. Hepatol Res. 2015;45(1):59-74.

5. Chen X, Liu HP, Li M, Qiao L. Advances in non-surgical management of primary liver cancer. World J Gastroenterol. 2014;20(44): 16630-16638.

6. Fonseca $\mathrm{AL}$, Cha $\mathrm{CH}$. Hepatocellular carcinoma: a comprehensive overview of surgical therapy. J Surg Oncol. 2014;110(6):712-719.

7. Lheureux S, Clarisse B, Launay-Vacher V, et al. Evaluation of current practice: management of chemotherapy-related toxicities. Anticancer Drugs. 2011;22(9):919-925.

8. Chen MH, Yang W, Yan K, et al. Large liver tumors: protocol for radiofrequency ablation and its clinical application in 110 patients mathematic model, overlapping mode, and electrode placement process. Radiology. 2004;232(1):260-271.

9. Huang YJ, Hsu HC, Wang CY, et al. The treatment responses in cases of radiation therapy to portal vein thrombosis in advanced hepatocellular carcinoma. Int J Radiat Oncol Biol Phys. 2009;73(4):1155-1163.

10. Marelli L, Stigliano R, Triantos C, et al. Treatment outcomes for hepatocellular carcinoma using chemoembolization in combination with other therapies. Cancer Treat Rev. 2006;32(8):594-606. 
11. Kamimura K, Suda T, Tamura Y, et al. Phase I study of miriplatin combined with transarterial chemotherapy using CDDP powder in patients with hepatocellular carcinoma. BMC Gastroenterol. 2012; $12: 127$.

12. Sahara S, Kawai N, Sato M, et al. Prospective evaluation of transcatheter arterial chemoembolization (TACE) with multiple anti-cancer drugs (epirubicin, cisplatin, mitomycin c, 5-fluorouracil) compared with TACE with epirubicin for treatment of hepatocellular carcinoma. Cardiovasc Intervent Radiol. 2012;35(6):1363-1371.

13. Onozuka H, Tsuchihara K, Esumi H. Hypoglycemic/hypoxic condition in vitro mimicking the tumor microenvironment markedly reduced the efficacy of anticancer drugs. Cancer Sci. 2011;102(5):975-982.

14. Gao Y, Shen JK, Milane L, Hornicek FJ, Amiji MM, Duan Z. Targeted cancer therapy: nanotechnology approaches for overcoming drug resistance. Curr Med Chem. 2015;22(11):1335-1347.

15. Shao RG, Zhen YS. Enediyne anticancer antibiotic lidamycin: chemistry, biology and pharmacology. Anticancer Agents Med Chem. 2008; $8(2): 123-131$

16. Shi YK, Wu SY, Huang YH, Zhen YS. Chemosensitivity of mdr1 gene overexpressed multidrug resistant cancer cells to lidamycin. Yao Xue Xие Bao. 2006;41(12):1146-1151. Chinese.

17. Beerman TA, Gawron LS, Shin S, Shen B, Mchugh MM. C-1027, a radiomimetic enediyne anticancer drug, preferentially targets hypoxic cells. Cancer Res. 2009;69(2):593-598.
18. Zhong G, Zhang S, Li Y, et al. A tandem scFv-based fusion protein and its enediyne-energized analogue show intensified therapeutic efficacy against lung carcinoma xenograft in athymic mice. Cancer Lett. 2010; 295(1):124-133.

19. Tang CM, Yu J. Hypoxia-inducible factor-1 as a therapeutic target in cancer. J Gastroenterol Hepatol. 2013;28(3):401-405.

20. Keith B, Johnson RS, Simon MC. HIF $1 \alpha$ and HIF2 $\alpha$ : sibling rivalry in hypoxic tumor growth and progression. Nat Rev Cancer. 2011; 12(1):9-22.

21. Martinengo C, Poggio T, Menotti M, et al. ALK-dependent control of hypoxia inducible factors mediates tumor growth and metastasis. Cancer Res. 2014;74(21):6094-6106.

22. Liao Y, Lu W, Che Q, et al. SHARP1 suppresses angiogenesis of endometrial cancer by decreasing hypoxia-inducible factor- $1 \alpha$ level. PLoS One. 2014;9(6):e99907.

23. Warfel NA, EI-Deiry WS. HIF-1 signaling in drug resistance to chemotherapy. Curr Med Chem. 2014;21(26):3021-3028.

24. Pires IM, Blokland NJ, Broos AW, et al. HIF-1 $\alpha$-independent hypoxiainduced rapid PTK6 stabilization is associated with increased motility and invasion. Cancer Biol Ther. 2014;15(10):1350-1357.

25. Wang T, Gikes DM, Takano N, et al. Hypoxia-inducible factors and RAB22A mediate formation of microvesicles that stimulate breast cancer invasion and metastasis. Proc Natl Acad Sci US A. 2014;111(31): E3234-E3242.
OncoTargets and Therapy

\section{Publish your work in this journal}

OncoTargets and Therapy is an international, peer-reviewed, open access journal focusing on the pathological basis of all cancers, potential targets for therapy and treatment protocols employed to improve the management of cancer patients. The journal also focuses on the impact of management programs and new therapeutic agents and protocols on

\section{Dovepress}

patient perspectives such as quality of life, adherence and satisfaction. The manuscript management system is completely online and includes a very quick and fair peer-review system, which is all easy to use. Visit http://www.dovepress.com/testimonials.php to read real quotes from published authors. 